\title{
OPTIMAL POWER GENERATION SCHEDULING OF A SHIPBOARD POWER SYSTEM
}

\author{
By: \\ W. Wu \\ D. Wang \\ A. Arapostathis \\ K. Davey
}

IEEE Electric Ship Technologies Symposium (ESTS 2007), Arlington, Virginia, U.S.A., May 21-23, 2007.

PN 319

Center for Electromechanics

The University of Texas at Austin

PRC, Mail Code R7000

Austin, TX 78712

(512) 471-4496 


\title{
Optimal Power Generation Scheduling of a Shipboard Power System
}

\author{
Wei $\mathrm{Wu}^{\dagger}$, Daifeng Wang ${ }^{\dagger}$, Ari Arapostathis ${ }^{\dagger}$ and Kent Davey ${ }^{\ddagger}$ \\ Department of Electrical and Computer Engineering ${ }^{\dagger}$ \\ and Center for Electromechanics ${ }^{\ddagger}$ \\ The University of Texas at Austin \\ E-mail: \{wuwei, dfwang, ari, k.davey\}@mail.utexas.edu
}

\begin{abstract}
This paper studies the optimal power generation scheduling for a shipboard power system. The problem is formulated as a discrete-time Markov Decision Process (MDP). The paper considers the shipboard power system both without any energy storage and with energy storage, and obtains an optimal power generation scheduling for each situation. For the non-storage system, the switch cost during the start-up process is considered. For the system with a storage, a fuel consumption comparison with different energy storage sizes is provided.
\end{abstract}

\section{INTRODUCTION}

The design and dynamic scheduling of turbine-generators plays a crucial role in the all-electric ship for the power density and efficiency. As the separation of prime-power sources supplying the propulsion loads and ship service loads is no longer necessary in the next generation electric ship, the granularity of the turbo-generator sets supplying the $80 \mathrm{MW}$ power capacity projected for $\mathrm{DD}(\mathrm{X})$-type ships and the dynamic scheduling of power generation according to demand are both subject to optimization. The choice of the set of turbogenerators and their respective generated power affects the overall power density, efficiency, operational characteristics and survivability of the whole system.

We aim to investigate the dynamic behaviors of power generation and find an optimal scheduling algorithm according to different mission profiles and system configurations.

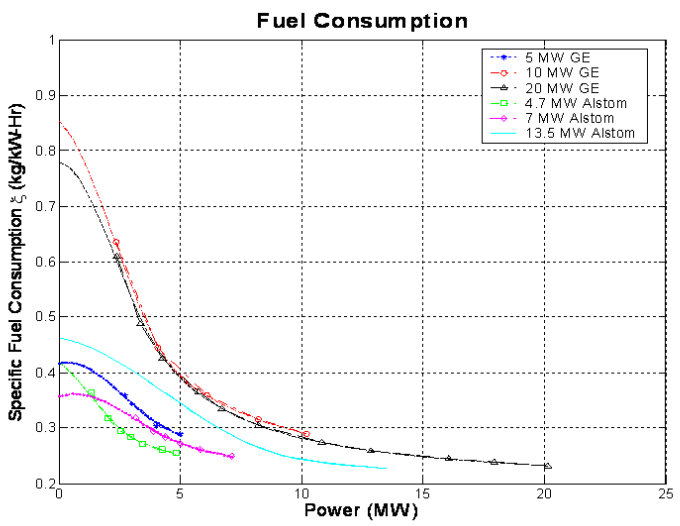

Fig. 1. Specific fuel consumption of seven commercial turbine-generators
Moreover, with various energy storage technologies available, it is imperative to understand their impact on power generation scheduling.

Figure I in [1] shows the specific fuel consumption for six commercial turbines. All turbines work most efficiently at peak load ratings, but lose considerable efficiency at partial power settings. Thus, intuitively, one should distribute the maximum load for some of turbines and keep others idle. On the other hand, switching turbines from OFF to $\mathrm{ON}$ requires additional fuel for the start-up process. Therefore there exists a tradeoff in balancing these two factors. Furthermore, by adding energy storage into the system, we can open up a new dimension for design and bring at least two advantages: first, it can reduce the frequency of turbines switching from OFF to ON thus reducing their overhead; second, it can allow turbines working mostly at peak load, the most efficient working point.

In this work, first, we formulate the dynamic generation scheduling of shipboard power systems as a controlled Markov decision process and study the optimal scheduling both analytically and numerically that maintains the level of energy storage and operates the turbine-generators so that the total fuel consumption is minimized subject to the dynamics of power loads. We then studies the shipboard power system with an energy storage. The dynamic programming is used to obtain the optimal control and the minimum average fuel consumption. We obtain the minimum average fuel consumption under different storage sizes for an $80 \mathrm{MW}$ destroyer on a four-day mission. We also analyze the optimal scheduling under two different settings that use different turbine-generators.

\section{Optimal Generation Scheduling Without ENERGY STORAGE: STATIC VS DYNAMIC}

We consider an electric ship with a number of $N$ turbinegenerators. The total power required for the ship at speed $v_{k}$ is $\bar{P}_{k}$. Let $P_{n, k}$ denote the power assigned to Generator $n$ at speed $v_{k}$. The specific fuel consumption of Generator $n$ denotes how much fuel is required to generate unit power at Generator $n$, and is typically an exponential function in $P_{n, k}$ (see Fig. I). Specifically,

$$
\xi_{n}\left(P_{n, k}\right)=\xi_{n, 0}+\frac{\xi_{n, 2}-\xi_{n, 0}}{1-e^{-m_{n}}}\left(1-e^{-\frac{P_{n, k}-P_{n, \min }}{P_{n, m a x}-P_{n, m i n}}}\right) .
$$


Fig. 2. Four 20.142 MW LM2500 turbine-generators Comparison of total fuel consumption in a 10-day task

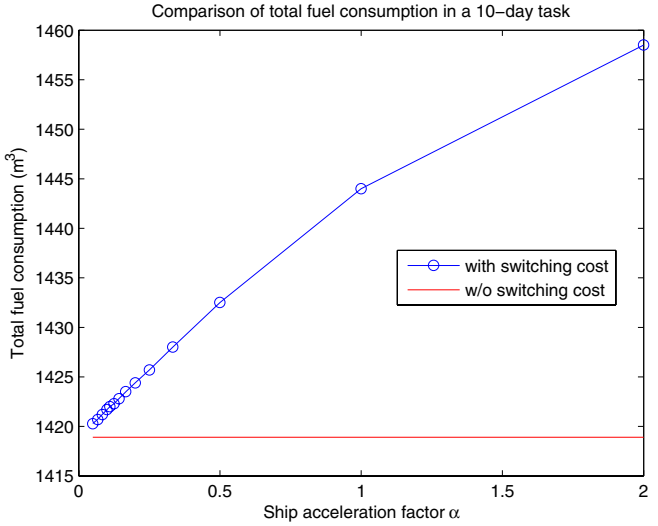

Thus, the fuel consumed at Generator $n$ is $P_{n, k} \xi_{n}\left(P_{n, k}\right)$. Given a mission profile, with $\eta_{k}$ denoting the sojourn time fraction at speed $v_{k}$ of a mission, generation scheduling can be formulated as the following optimization problem:

$$
\begin{array}{ll}
\text { minimize } & \sum_{k} \eta_{k} \sum_{n=1}^{N} P_{n, k} \xi_{n}\left(P_{n, k}\right) \\
\text { subject to } & \sum_{n=1}^{N} P_{n, k} \geq \bar{P}_{k} .
\end{array}
$$

However, the objective function in (2a) is non-convex, thus the optimal solution is computationally hard to obtain. We use a convex approximation of (2a) and obtain the approximate optimal solution using convex optimization.

However, the above formulation neglects the additional fuel consumption when generators switch from OFF to ON. We consider this effect by modeling the system as a Markov decision process (MDP), with state $\left(k_{t}, n_{t-1}\right)$ and action $\left(n_{t}, \mathbf{P}_{*, t}\right)$, where $n_{t}$ denotes the number of generators $\mathrm{ON}$ at time $t, k_{t}$ denotes the state of the ship speed, and $\mathbf{P}_{*, k_{t}}$ denotes the power vector $\left\{P_{i, t}, 1 \leq i \leq n_{t}\right\}$ at time $t$, $1 \leq k_{t} \leq K, 1 \leq n_{t} \leq N$. Under the assumption that the speed of ship changes according to an autonomous Markov chain, and denoting the startup fuel consumption for each generator $c_{\text {start }}$, the problem can be formulated as an MDP with state constraints:

minimize $\varlimsup_{T \rightarrow \infty} \frac{1}{T} \sum_{t=1}^{T}\left[\sum_{i=1}^{n_{t}} P_{i, t} \xi_{n}\left(P_{i, t}\right)+\left(n_{t}-n_{t-1}\right) c_{\text {start }}\right]$

subject to $\sum_{i=1}^{n_{t}} P_{i, t} \geq \bar{P}_{k_{t}}, \quad \forall t \in \mathbb{N}$.

Here we consider the infinite horizon long-term average cost and the optimal policy can be obtained by the value iteration algorithm in [2].

In Fig. 2 and Fig. 3, we compare the total fuel consumption under the optimal power generation scheduling policy between the case without startup fuel consumption (i.e. $c_{\text {start }}=0$ ) and the case with startup fuel consumption. In Fig. 2, four LM2500

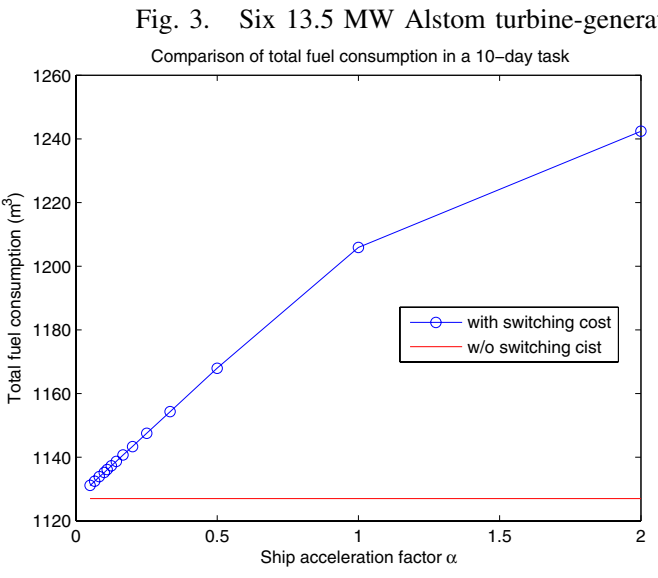

turbine-generators are used $(N=4)$ and we consider a family of Markov chains characterizing the speed changes of the ship, which have the same stationary distribution (or mission profile) but different transition rates (acceleration factor of the ship). As we can see, with the transition rates increasing, the additional fuel consumption due to the startup fuel penalty also increases and can possibly be as much as $40 \mathrm{~m}^{3}$, in certain cases. In Fig. 3, where six 13.5 MW Alston turbine-generators are used, the additional fuel consumption can be as much as $100 \mathrm{~m}^{3}$, which is nearly $10 \%$ more than the expected fuel consumption obtained by the static optimization in $(2 a)-(2 b)$.

The results obtained suggest the following:

- It is important to use power storage in electric ship to mitigate the impact of the startup fuel consumption and improve generation efficiency.

- We need to have control data of the ship acceleration distribution, and not only the mission profile, in order to be able to predict and accurately optimize the fuel consumption of generators.

- Adaptive optimal scheduling algorithms that tailor generation scheduling based on the most recent ship acceleration history would provide good solutions.

\section{Optimal CONTRol of EnERgy StOrage AND GENERATOR SCHEDULING}

In this section, we consider the optimal power generation scheduling with energy storage. We suppose that an $80 \mathrm{MW}$ destroyer on a four-day mission is equipped with four turbinegenerators. A reasonable energy storage is from $15 \mathrm{MJ}$ to $125 \mathrm{MJ}$ in the $80 \mathrm{MW}$ destroyer. The time slot unit is supposed to be minute. Thus, $1 \mathrm{MJ}$ is equal to $\frac{1}{60} \mathrm{MW}$-minute. The dynamic power demands of the ship are formulated as a discrete-time Markov chain with a finite state set $\boldsymbol{S}=$ $\{8,8.25,8.75, \ldots, 79.25,79.75,80\} \mathrm{MW}$; i.e., an arithmetic sequence with common difference $0.25 \mathrm{MW}$ from $8 \mathrm{MW}$ to 80 MW. The transition probability of this Markov chain is given by a random walk model as below:

$$
P\left(D_{k+1}=j \mid D_{k}=i\right)= \begin{cases}p_{i j} & \text { if }|j-i| \leq 1 \\ 0 & \text { else }\end{cases}
$$


TABLE I

SPECIFIC PARAMETERS FOR TURBINES

\begin{tabular}{|c|c|c|c|}
\hline Turbine & $\xi_{0}$ & $\xi_{2}$ & $\mathrm{~m}$ \\
\hline 5 MW GE & 0.344 & 0.29 & 1.5837 \\
20.142 MW GE LM2500 & 0.5232 & 0.231 & 4.8428 \\
50 MW RR MT50 & 0.5741 & 0.2 & 8.7273 \\
\hline
\end{tabular}

where $D_{k}$ is denoted as the power demand at time $k$ and $i, j \in \boldsymbol{S}$.

\section{A. Four similar turbine-generators}

First, we consider that all four turbine-generators are 20.142 MW GE LM2500 turbines. At the beginning of time $k$, we denote the stored energy as $E_{k}$, the power generated by each turbine-generator as $P_{k}$ and the number of the working turbine-generator as $N_{k} \in\{0,1,2,3,4\}$. Thus, the energy storage at time $k+1$ is given by

$$
E_{k+1}=E_{k}+N_{k} P_{k}-D_{k} .
$$

We need to decide $P_{k}$ and $N_{k}, k=0,1,2, \ldots, N-1$ to minimize the average fuel consumption over $N$ minutes. Therefore, the optimization problem can be formulated as an MDP as follows. The system state at time $k$ is described by $X_{k}=\left(E_{k}, D_{k}\right)$. The action is $U_{k}=\left(N_{k}, P_{k}\right)$, a twodimension control. Thus, given $X_{k}$ and $U_{k}$, the transition probability is described by

$$
\begin{aligned}
P\left(X_{k+1} \mid X_{k}, U_{k}\right) & =P\left(D_{k+1}, E_{k+1} \mid N_{k}, P_{k}, E_{k}, D_{k}\right) \\
& =P\left(D_{k+1} \mid D_{k}\right),
\end{aligned}
$$

where we assume that $D_{k+1}$ is independent of the action and the energy storage at time $k$.

The fuel consumption at time $k$ is the cost function at time $k$ given as below:

$$
\begin{aligned}
C\left(X_{k}, U_{k}\right) & =N_{k} P_{k} \xi\left(P_{k}\right) ; \\
\xi(P) & =\xi_{0}+\frac{\xi_{2}-\xi_{0}}{1-e^{-m}}\left(1-e^{-\frac{P-P_{m} a x}{P_{m} a x-P_{m} i n}}\right),
\end{aligned}
$$

where $\xi_{0}, \xi_{2}$ and $m$ can be found in Table I.

The optimal power generation scheduling comes up with $N$ optimal actions $U_{k}, k=0,1, \ldots, N$ such that it minimizes the average fuel consumption over $N$ minutes; i.e.,

$$
J_{0}^{*}\left(X_{0}\right)=\min _{U_{k}} \frac{1}{N} \sum_{k=0}^{N} C\left(X_{k}, U_{k}\right) .
$$

We solve this problem by the dynamic programming in [3] that is shown as follows:

$$
\begin{aligned}
J_{N}\left(X_{N}\right) & =0 ; \\
J_{k}\left(X_{k}\right) & =J_{k}\left(E_{k}, D_{k}\right) \\
& =\min _{N_{k}, P_{k}}\left\{C\left(X_{k}, U_{k}\right)\right. \\
& \left.+\sum J_{k+1}\left(E_{k}+N_{k} P_{k}-D_{k}, D_{k+1}\right) P\left(D_{k+1} \mid D_{k}\right)\right\} ; \\
J_{0}^{*}\left(X_{0}\right) & =\frac{J_{0}\left(X_{0}\right)}{N},
\end{aligned}
$$

which has a constraint, $E_{k}+N_{k} P_{k}-D_{k} \geq 0, k=0,1, \ldots, N-$ 1.

The simulation result for an $80 \mathrm{MW}$ destroyer on a four-day mission equipped with four 20.142 MW GE LM2500 turbinegenerators is shown in Fig. 4. As seen in Fig. 4, the average fuel consumption decreases as the energy storage increases. The maximum energy storage is $125 \mathrm{MJ}$ and consumes the less fuel per minute compared with the situation that we don't have any energy storage. Thus, an energy storage is indeed helpful to reduce the fuel consumption of the shipboard power system.

Fig. 4. Four 20.142 MW GE LM2500 turbine-generators

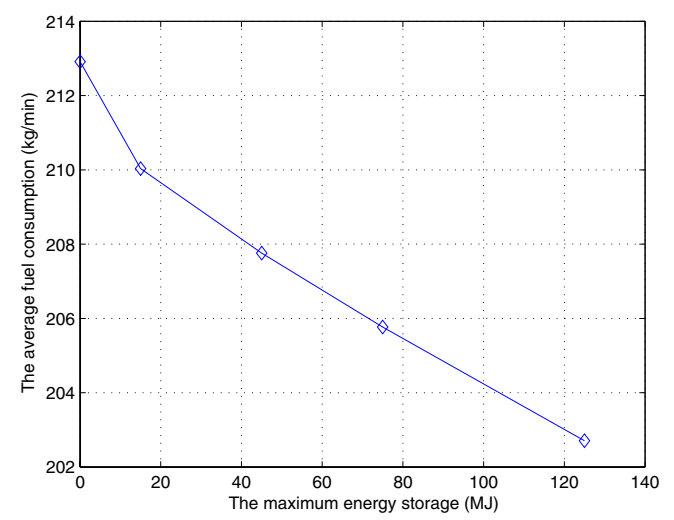

Fig. 5. Two $5 \mathrm{MW}$ GE and two $50 \mathrm{MW}$ RR MT50 turbine-generators

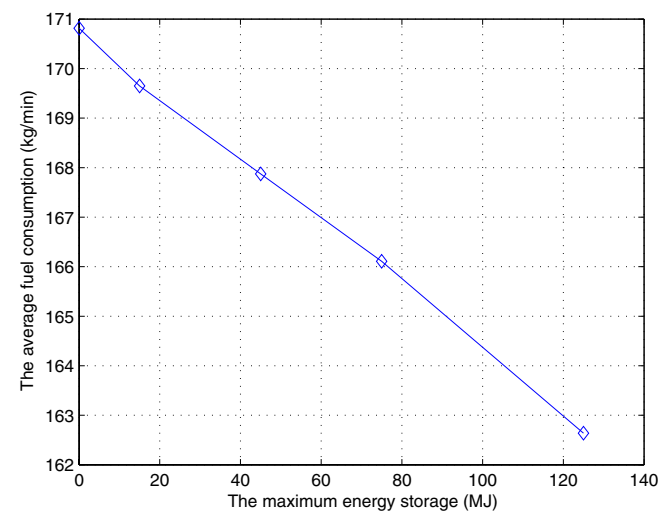

\section{B. Two small and two large turbine-generators}

Next, we consider that four turbine-generators are two 5 MW GE turbines and two 50 MW RR MT50 turbines. At the beginning of time $k$, we denote the stored energy still as $E_{k}$, the power offered by each $5 \mathrm{MW}$ generator as $P_{k}^{1}$, the number of the working $5 \mathrm{MW}$ generators as $N_{k}^{1} \in\{0,1,2\}$, the power offered by each $50 \mathrm{MW}$ generator as $P_{k}^{2}$, and the number of the working $50 \mathrm{MW}$ generators as $N_{k}^{2} \in\{0,1,2\}$. Thus, the stored energy at time $k+1$ becomes

$$
E_{k+1}=E_{k}+N_{k}^{1} P_{k}^{1}+N_{k}^{2} P_{k}^{2}-D_{k} .
$$


We also formulate the optimization problem as an MDP as follows. At time $k$, the system state is still $X_{k}=\left(E_{k}, D_{k}\right)$. Our action becomes a four dimensional control and given by $U_{k}=\left(N_{k}^{1}, N_{k}^{2}, P_{k}^{1}, P_{k}^{2}\right)$. Thus, the transition probability given the current state and its corresponding action is described by

$$
\begin{aligned}
P\left(X_{k+1} \mid X_{k}, U_{k}\right) & =P\left(D_{k+1}, E_{k+1} \mid N_{k}^{1}, N_{k}^{2}, P_{k}^{1}, P_{k}^{2}, E_{k}, D_{k}\right) \\
& =P\left(D_{k+1} \mid D_{k}\right) .
\end{aligned}
$$

Note that the assumption that $D_{k+1}$ is independent of the action and the energy storage at time $k$ still remains here.

The cost function at time $k$; i.e., the fuel consumption, is given by

$$
C\left(X_{k}, U_{k}\right)=N_{k}^{1} P_{k}^{1} \xi\left(P_{k}^{1}\right)+N_{k}^{2} P_{k}^{2} \xi\left(P_{k}^{2}\right)
$$

An optimal power generation scheduling minimizes the average cost-to-go fuel consumption over $N$ minutes, which is shown in (8).

The dynamic programming in [3] to solve the problem above is shown as follows:

$$
\begin{aligned}
J_{N}\left(X_{N}\right) & =0 ; \\
J_{k}\left(X_{k}\right) & =J_{k}\left(E_{k}, D_{k}\right) \\
& =\min _{N_{k}^{1}, N_{k}^{2}, P_{k}^{1}, P_{k}^{2}}\left\{C\left(X_{k}, U_{k}\right)\right. \\
& \left.+\sum_{k+1} J_{k}\left(E_{k}+N_{k}^{1} P_{k}^{1}+N_{k}^{2} P_{k}^{2}-D_{k}, D_{k+1}\right) P\left(D_{k+1} \mid D_{k}\right)\right\} ; \\
J_{0}^{*}\left(X_{0}\right) & =\frac{J_{0}\left(X_{0}\right)}{N},
\end{aligned}
$$

where we have a constraint, $E_{k}+N_{k}^{1} P_{k}^{1}+N_{k}^{2} P_{k}^{2}-D_{k} \geq 0$, $k=0,1, \ldots, N-1$.

The simulation result for an $80 \mathrm{MW}$ destroyer on a fourday mission equipped with two $5 \mathrm{MW}$ GE turbines and two 50 MW RR MT50 turbines is shown in Fig. 5. As seen in Fig. 5, the maximum energy storage of $125 \mathrm{MJ}$ reduces the fuel consumption per minute compared with the non-storage system. Moreover, the combination of these two small and two large turbine-generators also saves the certain amount of fuel per minute compared with using four similar turbine-generators. Thus, the different turbine-generators working together may also reduce the fuel consumption.

\section{Conclusion}

In our past work, we have studied the selection of both the number and the size of gas turbines by discrete optimization. Based on the optimal configuration we obtained, we consider the optimal generation scheduling problem to meet dynamic power demands of different mission profiles. We also model the power generation of an electric ship equipped with energy storage (i.e., flywheels) and study the optimal storage control and power generation problem. The problem is modeled as a discrete-time Markov decision process, whose optimal solution can be obtained by dynamic programming. An algorithm is developed to find the optimal scheduling for minimizing the long-term average fuel consumption. The impact of different system configurations is also investigated.

\section{REFERENCES}

[1] Kent Davey, "Ship Component in Hull Optimization," Marine Technology Society Journal, vol. 39, no. 2, pp. 42-49, Summer 2005.

[2] A. Arapostathis, V. S. Borkar, E. Fernandez-Gaucherand, M. K. Ghosh and S. I. Marcus, "Discrete-Time Controlled Markov Processes with Average Cost Criterion: A Survey," SIAM J. Control and Optimization, vol. 31, no. 2, pp. 282-344, March 1993.

[13 Dimitri P. Bertsekas, Dynamic Programming and Optimal Control, vol. I., Athena Scientific, MA, USA, 2001. 